\title{
ESQUEMA PRÁCTICO DEL MANEJO DE LOS EFECTOS ADVERSOS ASOCIADOS CON LA INSTILACION DE BCG
}

\author{
Fernando Rodríguez, Juan Palou, R. Martínez, O. Rodríguez, A. Rosales, Jorge Huguet y \\ Humberto Villavicencio.
}

Servicio de Urología. Fundació Puigvert. Barcelona. España.

\begin{abstract}
Resumen.- OBJETIVOS: La morbilidad secundaria al tratamiento con Bacilo de Calmette-Guèrin (BCG) intravesical puede presentarse tanto local como sistémicamente. La gran mayoría de los pacientes experimentan un síndrome miccional irritativo autolimitado. A menudo no existen criterios unificados sobre el manejo de los efectos secundarios a la BCG. Después del tratamiento de más de 500 pacientes con BCG hemos desarrollado un esquema práctico en el manejo de su morbilidad. Presentamos de una forma esquemática y clara, la pauta que seguimos en nuestro centro cuando un paciente presenta síntomas y morbilidad secundaria a las instilaciones intravesicales con BCG.
\end{abstract}

MÉTODOS: Se analiza y describe, según la bibliografía y experiencia propia, el manejo de los efectos adversos experimentados por los pacientes tratados con BCG intravesical, desde que se inició de forma protocolizada su indicación en pacientes con tumor vesical no músculo invasivo de alto grado y/o CIS.

RESULTADOS: Dentro de los síntomas mas frecuentes están los síntomas miccionales imitativos, generalmente autolimitados; pero que su persistencia (>48 horas), deben llevar al urólogo a su tratamiento según intensidad y duración. La macrohematuria no es infrecuente y cede con conducta expectante e ingesta hídrica. Pero también puede ser una infección urinaria o un tumor residual. El síndrome febril, si presente, habitualmente se autolimita en las primeras 24-48 horas siendo inferior a $38.5^{\circ} \mathrm{C}$ sin afectación del estado general. En los casos de persistencia y/o sepsis se deberá iniciar tratamiento con tuberculostáticos y/o corticoides. Pueden aparecer otros cuadros como la orquiepididimitis, artritis, etc.

CONCLUSIONES: El diagnóstico y tratamiento adecuado de los efectos adversos de la BCG, es básico para permitir que la immunoterapia intravesical sea prescrita y manejada correctamente por los urólogos, y permita un tratamiento adecuado de los pacientes evitando la posibilidad de complicaciones más severas.

Palabras clave: BCG. Terapia intravesical. Efectos adversos.

Summary.- OBJECTIVES: Morbidity secondary to intravesical Bacillus Calmette-Guèrin (BCG) may present both locally and systemically. Most patients suffer a selflimited irritative voiding syndrome. Often, there are not unified criteria for the management of BCG side effects. After treating more than 500 patients with BCG we developed a practical guideline for the management of its morbidity. 
We present clearly and schematically the practice guideline we follow in our Center when the patient presents symptoms and morbidity secondary to BCG intravesical installations.

METHODS: We analyze and describe, following the literature and our own experience, the management of adverse events experienced by patients treated with intravesical BCG, since the initial implementation of the protocol for its indication in patients with high risk nonmuscle invasive bladder tumors and/or CIS.

RESULTS: Irritative voiding symptoms are among the most frequent symptoms, generally self-limited; but if they persist ( $>48$ hours) will have the urologist treat them depending on intensity and duration. Macroscopic hematuria is not unfrequent and diminishes with an expectant approach and water intake. But, it may also be a urinary tract infection or residual tumor. A febrile syndrome, if present, is usually self-limited to the first 24-48 hours and below $38.5^{\circ} \mathrm{C}$ without general status affectation. In cases of persistence and/or sepsis, tuberculostatic treatment and/or corticoids should be started. Other clinical pictures may appear, such as orchyoepididymitis, arthritis, etc.

CONCLUSIONS: Proper diagnosis and treatment of adverse events after BCG therapy are basic to allow intravesical immunotherapy be properly prescribed and managed by urologists, enabling a proper treatment of patients and avoiding the possibility of more severe complications.

Keywords: BCG. Intravesical therapy. Adverse events.

\section{MANEJO DE LOS EFECTOS ADVERSOS DE LA BCG}

El efecto toxico de las instilaciones intravesicales con Bacilo de Calmette-Guèrin (BCG) puede presentarse tanto local como sistémicamente. La mayoría de los pacientes experimentan un síndrome miccional irritativo autolimitado. Generalmente se presenta en las primeras cuatro horas posteriores a la instilación, desapareciendo habitualmente en las siguientes 48 horas sin tratamiento. En algunos casos puede prolongarse días o semanas (Figura 1) $(1,2)$. Las manifestaciones generales son las propias de una respuesta inflamatoria sistémica, apareciendo en un periodo de tiempo similar y caracterizándose por fiebre, escalofríos e incluso artralgias. La febrícula y la sensación de malestar leve pueden afectar a un porcentaje significativo de pacientes. La presencia de fiebre mayor de 38,5 grados centígrados que persiste durante más de 24 horas y no remite con agentes antipiréticos, o de fiebre mayor de 39,5 grados centígrados requiere tratamiento tuberculostático* (Figura 2) (1).

No es infrecuente una artralgia reactiva posterior a instilaciones intravesicales de BCG. Se trata de una complicación usualmente bien controlada con la suspensión de la inmunoterapia y la administración de un AINE (Figura 3). Algún paciente llega a desarrollar una artritis, un proceso más agudo y severo que requiere tratamiento tubeculostático* y corticoides (3). Es posible que durante alguna de las instilaciones del ciclo de mantenimiento, cualquiera de los síntomas mencionados anteriormente se vuelva a presentar, en general de forma leve. La mayoría responden al tratamiento con un AINE.

El tratamiento con BCG también puede inducir la aparición de hematuria en un 30\% de los pacientes (4). La hematuria macroscópica persistente es una contraindicación relativa para el tratamiento; es aconsejable suspender el tratamiento hasta su resolución. Si ésta es persistente, en ausencia de infección urinaria, se aconseja practicar cistoscopia para descartar persistencia de enfermedad tumoral en vejiga (Figura 4).

La aparición de tumefacción escrotal con enrojecimiento local y engrosamiento del epidídimo sugiere una orquiepididimitis. Si se trata de un cuadro agudo y de presentación inmediata a las instilaciones inicialmente se administrará una fluoquinolona, ya que habitualmente se trata de una infección bacteriana. Si no responde al tratamiento y/o el cultivo de orina es negativo, se administraran tuberculostaticos* (Figura 5). Si se presenta de forma tardía, entonces debe tratarse inicialmente con tuberculostáticos*, ya que la causa más frecuente es entonces la infección microbacteriana (5).

Se podría encontrar algún caso donde exista el paso de la BCG al torrente sanguíneo resultando en un proceso mucho mas grave y con síntomas severos, se denomina BCGosis (6); ésta se caracteriza por ser un proceso de evolución mucho mas violento y rápido que aparece dentro de las dos primeras horas post-instilación o incluso inmediatamente después de un cateterismo traumático. En estos casos se debe iniciar de forma inmediata el tratamiento con tuberculostáticos* y corticoides a altas dosis.

Episodios febriles que comiencen después de 24 horas de la instilación y que persistan mas de 48 horas son indicativos de infección por la propia manipulación (infección bacteriana) o por la propia 


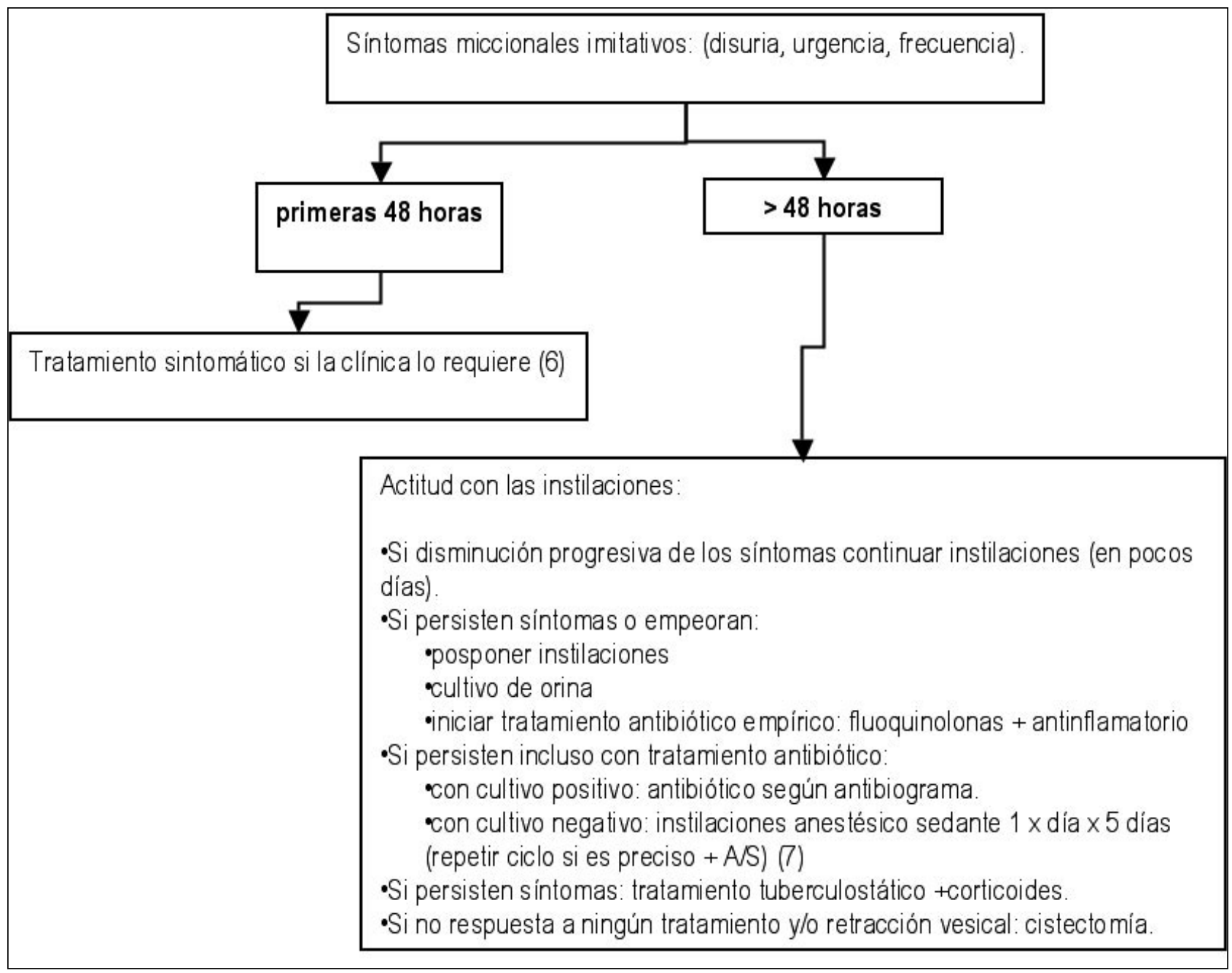

FIGURA 1.

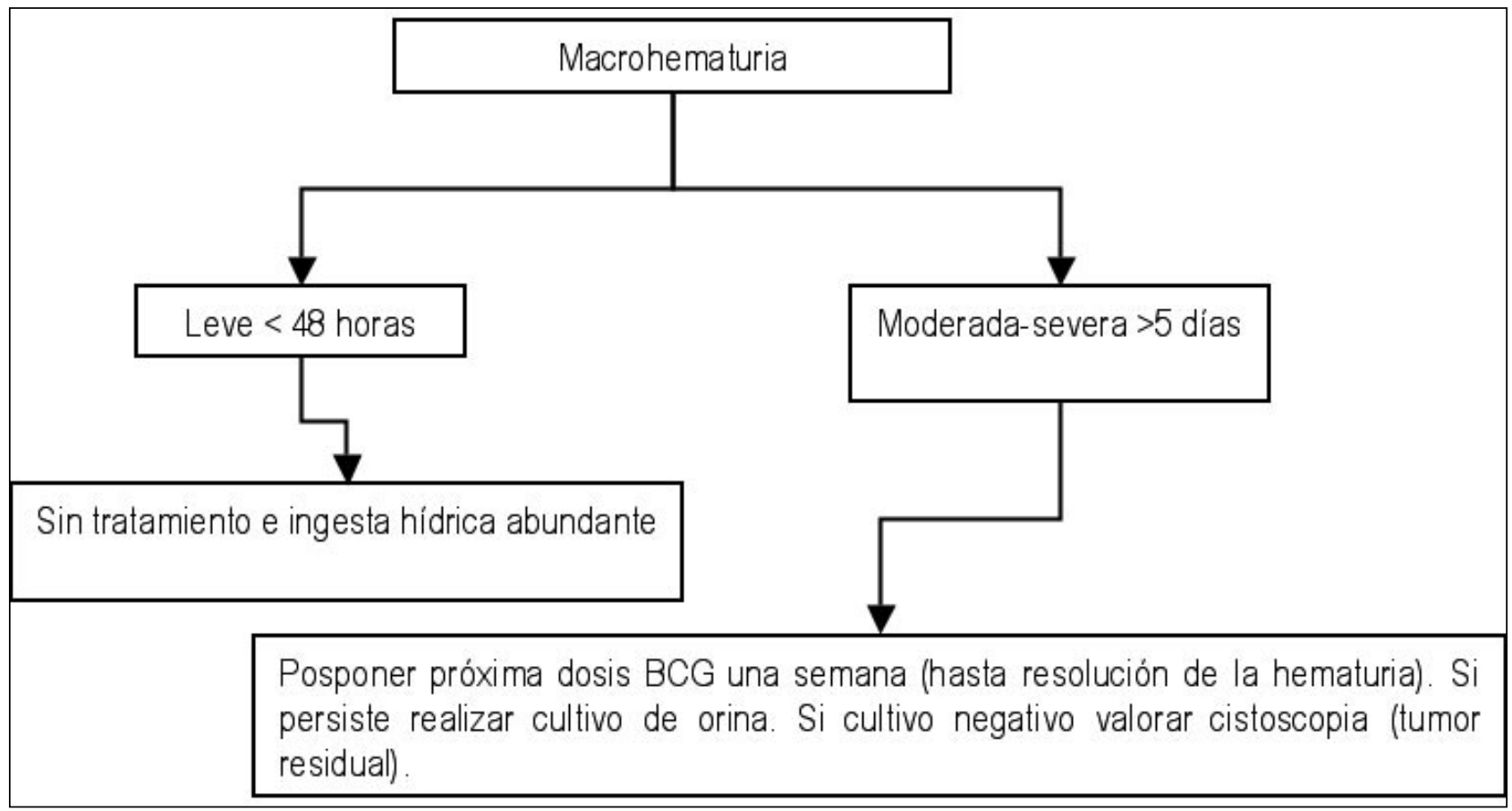

FIGURA 2. 


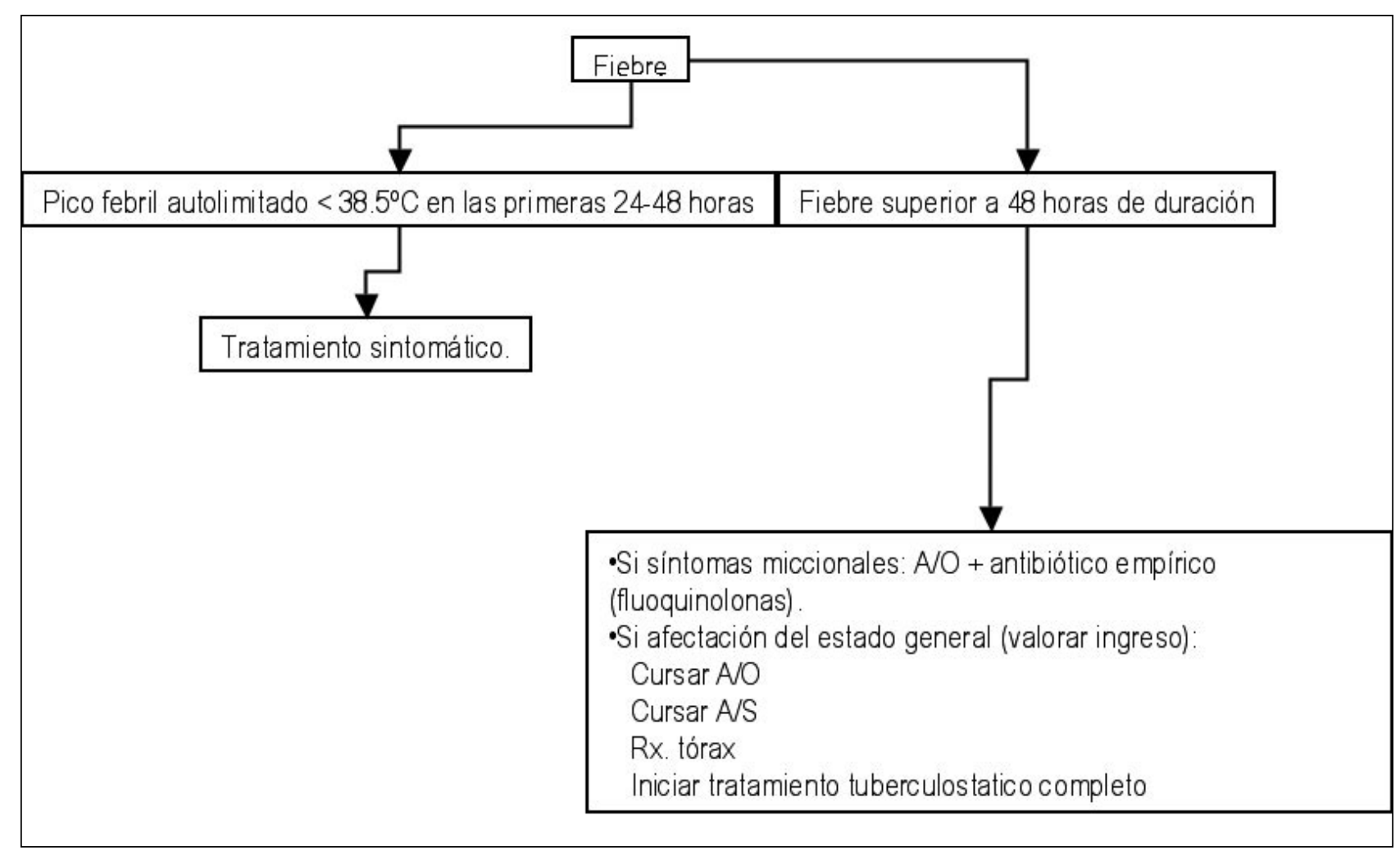

FIGURA 3.

BCG (BCGitis) (7). Estos pacientes deben ser hospitalizados e iniciar tratamiento antibiótico empírico y luego continuar dependiendo del resultado de los cultivos y la severidad del cuadro.
La pauta a seguir según la morbilidad por las instilaciones variara en función de cada una y de su gravedad; puede ser desde posponer las instilaciones, disminuir la dosis de las mismas o suspenderlas (Tabla I) (8).

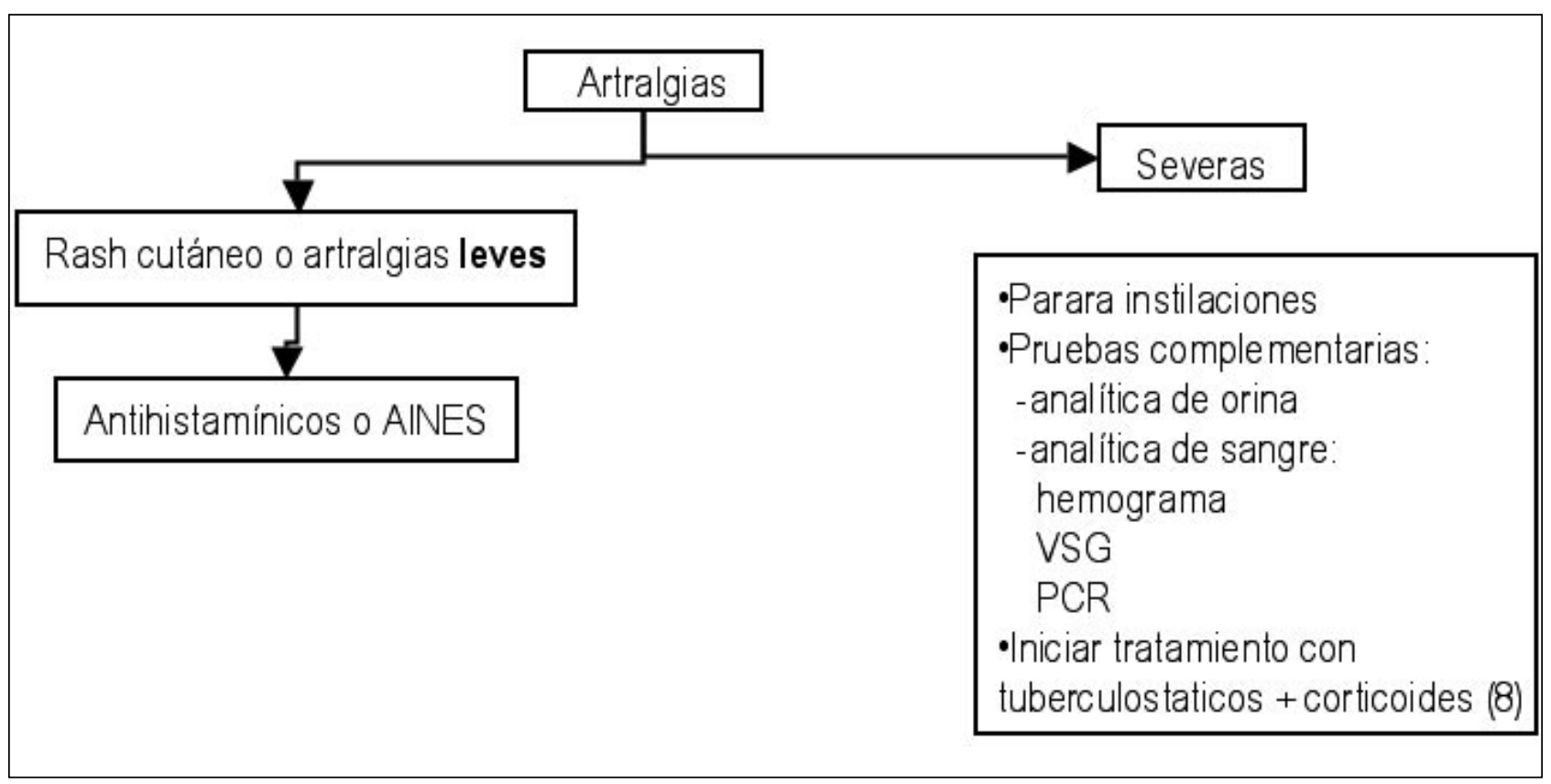




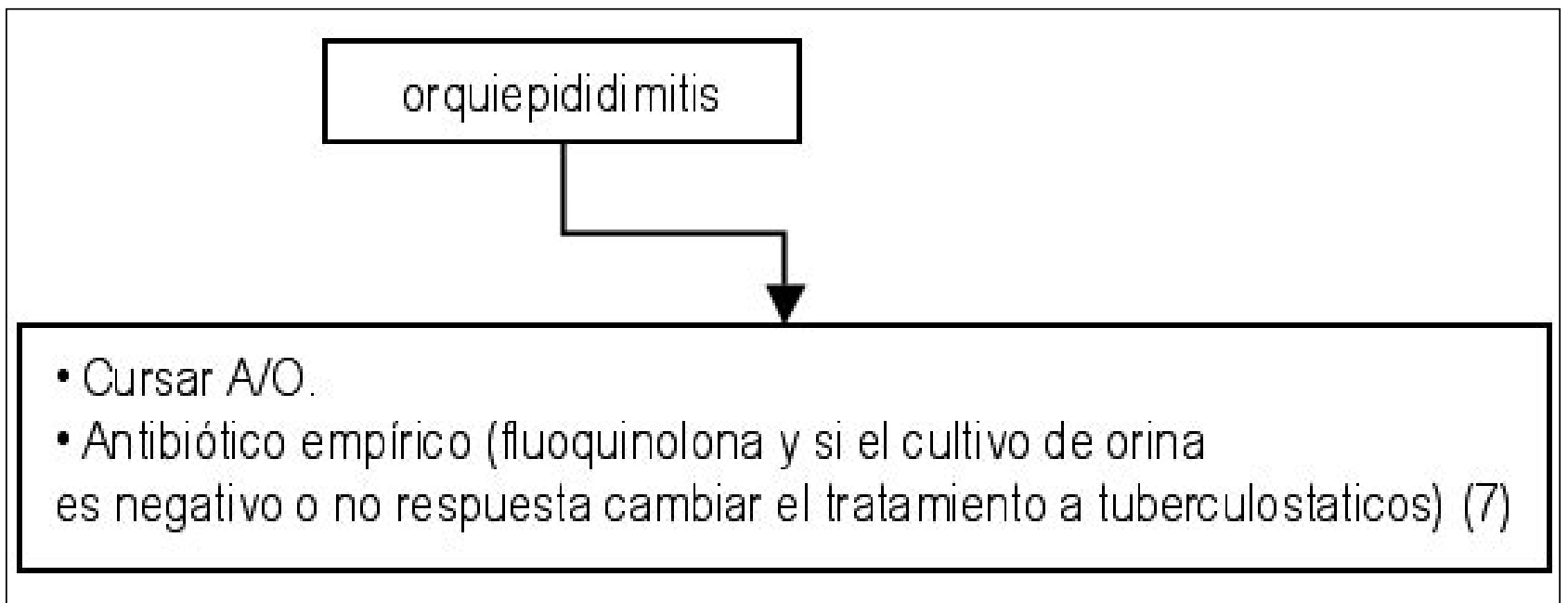

\section{FIGURA5.}

Globalmente el tratamiento con BCG esta contraindicado en paciente con:

1. TBC activa por peligro de exacerbación o una reacción sistémica BCG concomitante.

2. Evidencia previa o actual de una reacción sistémica a la BCG.
3. Fiebre, a no ser que la causa de la fiebre este filiada.

4. Infección bacteriana del tracto urinario no tratada.

5. Cateterización traumática.

6. Tratamiento inmunosupresor (aunque se han des-

TABLA I. PAUTA A SEGUIR SEGÚN LA COMPLICACIONES (MODIFICADO DE PALOU (9).

\begin{tabular}{|l|l|}
\hline Complicación & Instilaciones \\
\hline Cistitis $<48$ horas & Continuar \\
\hline Cistitis severa ó $>4$ días & Posponer / Disminuir dosis / Suspender \\
\hline Fiebre $<48$ horas & Continuar \\
\hline Fiebre $>48$ horas & Posponer / Suspender \\
\hline Sepsis por Mycobacteria & Suspender \\
\hline Hematuria & Posponer \\
\hline Orquitis & Suspender \\
\hline Prostatitis granulomatosa sintomática & Posponer \\
\hline Artralgias & Posponer / Disminuir dosis \\
\hline Artritis severa & Suspender \\
\hline
\end{tabular}


crito casos de administración de BCG en pacientes con trasplante renal, con buenos resultados y sin morbilidad secundaria) (9).

En la actualidad se pueden utilizar las fluoquinolonas como tratamiento tuberculostático en el manejo de los efectos adversos (leves-moderados) del tratamiento con BCG (10). Es útil como fármaco de segunda línea en tuberculosis humana e incluso se ha llegado a demostrar en modelos animales que el uso de este antibiótico no afecta la eficacia antitumoral de la BCG (1 1).

\section{CONCLUSIÓN}

El diagnóstico y tratamiento adecuado de los efectos adversos de la BCG, es básico para permitir que la immunoterapia intravesical sea prescrita y manejada correctamente por los urólogos, y permita un tratamiento adecuado de los pacientes evitando la posibilidad de complicaciones más severas.

\section{BIBLIOGRAFÍA y LECTURAS

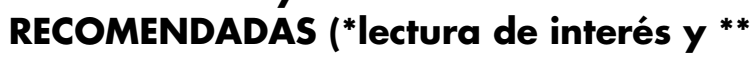 lectura fundamental)}

**1. LAMM, D.; STOGDILL, V.; BRIAN, J. y cols.: "Complications of bacilus Calmete-Guerin immunotherapy in 1278 patients with bladder cancer". J. Urol., 135: 272, 1986.

*2. PALOU, J.; RODRÍGUEZ-VILLAMIL, L.; ANDREU CRESPO, A. y cols.: "Intravesical treatment of severe bacilus Calmette-Guerin cystitis". Int. Urol. Nephrol., 33: 485, 2001.
3. TINAZZI, E. y cols.: "Reactive arthritis following BCG immunotherapy for urinary bladder carcinoma: A systematic review". Rheumatol. Int., 12: 1, 2005.

4. PATRIKC, C.; WALSH. y cols.: "Campbell: Urología". $8^{\mathrm{a}}$ ed. Tomo 4. Medica Panamericana. Buenos Aires-Argentina 2004.

5. FALKENSAMMER, C. y cols.: "Late occurrence of bilateral tuberculous-like epididymo-orchitis after intravesical bacille Calmette-Guerin therapy for superficial bladder carcinoma". Urology, 65: 175, 2005.

6. O’DONNELL, J.; BURNS, A.: "Intravesical Basile Calmette-Guérin therapy in the treatment and prophylaxis of urothelial bladder cancer. In Urothelial Tumors. Michael J. Droller. Pgs:219-247. Ed. BC Decker INC. London 2004.

7. RAWLS, W.; LAMM D.; LOWE, B. y cols.: "Fatal synopsis following intravesical bacilus Calmette-Guerin administration for bladder cancer". J. Urol., 144: 1328, 1990.

8. PALOU, J.: "Inmunoterapia en el carcinoma transicional superficial de vejiga". Tumores vesicales superficiales por J Vicente, G Chechile y J Salvador. pag. 175-189. Ed. Accion Medica. Barcelona, 2000.

9. PALOU, J.; ANGERRI, O.; SEGARRA, J. y cols.: "Intravesical bacilus Calmette-Guerin for the treatment of superficial bladder cancer in renal transplant patients". Transplantation., 76: 1514, 2003.

*10. FENDLER, J.P.: "Can ofloxacin constitute an alternative antitubercular treatment for BCG hepatitis?". Prog. Urol., 12: 309, 2002.

*11. DUREK, C.; JURCZOK, A.; WERNER, H. y cols.: "Optimal treatment of systemic bacillus Calmette-Guérin infection: Investigations in an animal model”. J. Urol., 168: 826, 2002. 